\title{
Tratamiento Fisicoquímico de las Aguas Residuales Generadas en el Proceso de Beneficio de Arcillas y Alternativas de Uso de los Lodos Generados en el Proceso
}

\author{
Biviana A. Llano, Jhon F. Cardona, David Ocampo y Luis A. Ríos \\ Grupo Procesos Químicos Industriales, Facultad de Ingeniería, Universidad de Antioquia, UdeA, Calle 70 \\ No.52-21, Medellín-Colombia (e-mail: bivianaastrid@gmail.com, fredycardona@gmail.com, \\ davidocampoe@gmail.com, lariospfa@gmail.com)
}

Recibido Nov. 7, 2013; Aceptado Ene. 8, 2014; Versión final recibida Ene. 28, 2014

\begin{abstract}
Resumen
El tratamiento fisicoquímico de las aguas residuales provenientes de una industria local de beneficio de arcillas para la remoción de sólidos suspendidos totales (SST) y turbiedad, utilizando la prueba de jarras ha sido estudiado y analizado. Adicionalmente, se caracterizó la materia prima utilizada en el proceso de beneficio, al igual que los lodos generados en el mismo. Los coagulantes utilizados fueron sulfato de aluminio y un polímero aniónico (A-100). Se evaluaron dos niveles de $\mathrm{pH}(6$ y 9 ) y dos dosis de coagulante $(20 \mathrm{mg} / \mathrm{L}$ y $100 \mathrm{mg} / \mathrm{L})$. Los resultados indican que con una baja concentración de coagulante es posible obtener una alta remoción cercana al 100\% de ambos parámetros, SST y turbiedad. El polímero aniónico mostró ser altamente eficaz en concentración de $20 \mathrm{mg} / \mathrm{L} \mathrm{y} \mathrm{pH}=6$. De acuerdo a los resultados obtenidos se propone una serie de aplicaciones en las cuales este material puede ser reutilizado.
\end{abstract}

Palabras clave: aguas residuales, arcillas, caolines, tratamiento fisicoquímico, lodos residuales

\section{Physical-Chemical Treatment of Wastewater from the Benefit Process of Clays and Alternative uses of the Sludge Generated by the Process}

\begin{abstract}
The physical-chemical treatment of wastewater from a local industry of benefit of clays by means of jar test for the removal of total suspended solids (TSS) and turbidity has been studied and analyzed. Also, the characterization of the raw material and the residual sludge used in the benefit process was done. The coagulants used were aluminum sulfate and an anionic polymer (A-100). Two pH levels (6 and 9) and two doses of coagulant $(20 \mathrm{mg} / \mathrm{L}$ and $100 \mathrm{mg} / \mathrm{L})$ were evaluated. The results showed that with a low coagulant concentration it is possible to obtain a high removal, close to $100 \%$, of TSS and turbidity. The anionic polymer showed to be highly effective at concentration of $20 \mathrm{mg} / \mathrm{L}$ and $\mathrm{pH}=6$. According to the results, several applications, where the sludge can be reused, were proposed.
\end{abstract}




\section{INTRODUCCIÓN}

El conjunto de actividades del ser humano es, en esencia, una fuente de desechos. Específicamente en centros urbanos, las aguas residuales provenientes de fuentes domésticas e industriales pueden ser nocivas (Beltrán-Heredia y Sánchez-Martín, 2009) y deben ser tratadas adecuadamente con el fin de evitar riesgos para el medio ambiente y la salud (Koivunen et al., 2003). El acelerado desarrollo industrial ha conducido a un deterioro ambiental de los recursos hídricos en el mundo y al surgimiento de problemas sanitarios y enfermedades asociadas al consumo de agua que afectan a comunidades enteras. Se estima que el $80 \%$ de las enfermedades existentes en países en vía de desarrollo son causadas por un abastecimiento de agua deficiente (World Health Organization, 2012).

En la actualidad, es alarmante el incremento de la explotación minera, ya que esta hace uso de grandes cantidades de agua para el procesamiento de los minerales, lo cual genera efluentes cargados en metales y sólidos, influyendo negativamente en el sano desarrollo de las comunidades aledañas a estos procesos (Kim et al., 2011; Ji et al., 2013). Las enfermedades diarreicas son la segunda mayor causa de muerte de niños menores de 5 años, ocasionando alrededor de 1.5 millones de muertes de infantes cada año. Se registran aproximadamente 2000 millones de casos por año, siendo la principal causa de malnutrición; adicionalmente la exposición a concentraciones elevadas de metales pesados, como el mercurio y plomo, causa múltiples problemas en órganos vitales, siendo la población más vulnerable los niños y las comunidades que consumen alimentos que tengan contacto directo con estos efluentes contaminados (World Health Organization, 2012). Estos fenómenos tienen una marcada incidencia en países subdesarrollados.

En este contexto, se ha establecido que el sector industrial no sólo es el que más agua demanda, sino también el que contamina en mayor medida este recurso. Un tratamiento adecuado de las aguas residuales industriales es de vital importancia para garantizar la disponibilidad a largo plazo del recurso agua y para permitir la reutilización del mismo en múltiples usos al interior de las industrias que lo contaminan, máxime si se tiene en cuenta que el agua es una materia prima crítica para la industria. Colombia, país que posee una gran riqueza hídrica disponible como agua superficial, ocupando el cuarto lugar a nivel mundial, presenta como principal problema medioambiental, después de la deforestación y la erosión del suelo, la contaminación del agua. En el año 1999, el 97\% de las aguas residuales se vertían a los ríos sin ningún tratamiento, lo cual ha conducido al deterioro paulatino de los recursos hídricos por la descarga de aguas residuales de origen doméstico, industrial, hospitalario, agroquímico, derrames de petróleo y desechos peligrosos (Alvarez, et al., 2001).

Específicamente, la industria cerámica vierte aguas residuales que presentan turbidez y color debido a la presencia de partículas muy finas suspendidas de materiales arcillosos. Desde el punto de vista químico, estas aguas se caracterizan por la presencia de sólidos suspendidos (arcillas, silicatos), aniones disueltos, metales pesados disueltos y suspendidos $(\mathrm{Pb}, \mathrm{Zn})$, boro en pequeñas cantidades y trazas de materia orgánica (Environmental Protection Agency, 2012). Igualmente producen grandes cantidades de lodos residuales que usualmente son desechados.

Las aguas residuales son comúnmente tratadas mediante procesos biológicos. No obstante, en el caso específico de las aguas residuales industriales, los procesos fisicoquímicos son una alternativa viable, eficiente y económica para tratarlas (Aragonés-Beltrán et al., 2009; Li et al., 2013). El proceso de coagulación-floculación es una etapa importante en las plantas potabilizadoras de agua, siendo utilizado en más del $95 \%$ de ellas. La predicción de la dosis óptima de coagulante es un factor crucial, ya que las regulaciones existentes en cuanto a los niveles residuales de coagulantes en aguas distribuidas son cada vez más estrictas (Bouyer et al., 2005). En este sentido, la prueba de jarras es el método comúnmente empleado para determinar la dosis apropiada de coagulante que debe utilizarse, en orden de garantizar una presencia mínima de sales de aluminio o de hierro residuales (Clark y Stephenson, 2009; Barrera-Díaz et al., 2009). Los procesos de coagulación son aptos no solo para remover partículas, también son capaces de remover patógenos que se encuentran unidos a dichas partículas, mejorando significativamente la calidad del agua y, en consecuencia, la salud humana (Miller et al., 2008; McCarthy y Zachara, 1989).

La economía mundial actual demanda una gran productividad en los diferentes mercados, ocasionando una rápida disminución de los recursos naturales disponibles y, simultáneamente, la generación de grandes volúmenes de residuos, los cuales, en su mayoría, no están siendo reciclados o reincorporados al proceso. En este sentido, el sector industrial ha llegado a ser considerado como una importante fuente de contaminación ambiental, dados los grandes volúmenes de materias primas que procesa y la cantidad de desechos que genera. La extracción, y el consecuente procesamiento de minerales, son un buen ejemplo de esta situación (Rauup-Pereira et al., 2006). El caolín es una materia prima fundamental en diferentes industrias tales como la cerámica, plásticos, caucho, tinta, cemento, químicos y la industria papelera. En 
muchos países, la mayoría de los desechos generados por las industrias de beneficio de caolín son descargados en corrientes de agua y ríos o vertidos en zonas al aire libre, afectando dramáticamente los ecosistemas (suelo, agua, fauna, flora) y la salud de las comunidades aledañas (Menezes et al., 2008).

Es claro que deben investigarse tecnologías de reutilización o de reciclaje apropiadas para los residuos generados (Souza y Mansur, 2004) (Moreno et al., 2006), contribuyendo de esta forma a la conservación de los recursos naturales, a incrementar la productividad de los procesos disminuyendo sus costos y a proporcionar materias primas alternativas para una gran variedad de procesos industriales. En este trabajo se presenta la evaluación del proceso de coagulación-floculación a nivel de laboratorio para las aguas residuales de una industria local de beneficio de arcillas y caolines, por medio de la prueba de jarras. Igualmente, se caracterizó la materia prima y los lodos generados en el proceso industrial, en orden de discernir la posibilidad de reutilizar este material en diferentes aplicaciones.

\section{METODOLOGÍA}

Se efectuó un proceso de toma de muestra compuesto (volumen total $5 \mathrm{~L}$ ), el cual consistió en tomar porciones en un intervalo de tiempo definido $(30 \mathrm{~min})$, teniendo en cuenta el caudal instantáneo por el método de aforo volumétrico, durante un tiempo prolongado (4 horas) de acuerdo a la norma ASTM D3370, con el fin de obtener una muestra representativa de efluentes residuales, en una industria del sector cerámico local. Estos efluentes fueron caracterizados fisicoquímicamente, determinando los parámetros: SST (sólidos suspendidos totales), turbiedad reposada, turbiedad agitada, conductividad, DBO5 y DQO. Paralelamente, se caracterizaron las tres arcillas utilizadas por la empresa como materia prima M1, M2 y M3 y además se obtuvo una muestra representativa de lodos residuales del proceso industrial, la cual consistía en una mezcla de todos los lodos generados por la empresa durante 3 días de operación de la planta. Dicha caracterización se efectuó con el fin de comparar la composición química del lodo residual con la composición de las materias primas, y de esta forma establecer y sugerir alternativas de valorización de los lodos generados en el proceso de beneficio de la empresa.

El proceso de coagulación-floculación se llevó a cabo a nivel de laboratorio siguiendo la metodología de la prueba de jarras (Aragonés-Beltrán et al, 2009) (Guan et al, 2005), utilizando $1 \mathrm{~L}$ de muestra en cada recipiente. Se evaluó un diseño experimental factorial $2^{3}$ de tres variables con dos niveles (Ver Tabla 1): dos niveles de $\mathrm{pH}, 6$ y 9 , dos dosis de coagulante, $20 \mathrm{mg} / \mathrm{L}$ y $100 \mathrm{mg} / \mathrm{L}$, y dos tipos de coagulante, sulfato de aluminio y el polímero aniónico A-100, con el fin de determinar la eficiencia de remoción del proceso. El polímero aniónico utilizado es el producto comercial SUPERFLOC A-100 HMW (Kemira), cuyo carácter químico corresponde a una poliacrilamida aniónica de alto peso molecular. Puede ser utilizado como ayudante de la coagulación y a bajas dosis presenta una alta efectividad que permite reducir o eliminar el uso de sales inorgánicas. El sulfato de aluminio corresponde al producto comercial Kemira ALC 2-5, libre de hierro, basado en aluminio trivalente. Es un coagulante eficiente para el tratamiento de agua potable y residual, tanto municipal como industrial.

Tabla 1: Diseño de experimentos prueba de jarras.

\begin{tabular}{|c|c|c|l|}
\hline Experimento & $\mathrm{pH}$ & $\begin{array}{c}\text { Concentración } \\
\text { coagulante }(\mathrm{mg} / \mathrm{L})\end{array}$ & \multicolumn{1}{|c|}{ Tipo coagulante } \\
\hline 1 & 9 & 100 & Sulfato aluminio \\
\hline 2 & 9 & 20 & Sulfato aluminio \\
\hline 3 & 6 & 100 & Polímero \\
\hline 4 & 6 & 20 & Polímero \\
\hline 5 & 9 & 100 & Polímero \\
\hline 6 & 9 & 20 & Polímero \\
\hline 7 & 6 & 100 & Sulfato aluminio \\
\hline 8 & 6 & 20 & Sulfato aluminio \\
\hline
\end{tabular}

La metodología incluyó un ajuste inicial de pH del agua residual a 6.0 utilizando una solución de ácido sulfúrico $1 \mathrm{~N}$ y el ajuste a pH 9 con una solución de hidróxido de sodio $1 \mathrm{~N}$. Una vez ajustado el pH, el agua se dejó bajo agitación a $120 \mathrm{rpm}$ y se adicionó la cantidad requerida de coagulante, permitiendo un mezclado rápido de 2 minutos de duración, seguido por un mezclado lento a $40 \mathrm{rpm}$ durante 30 minutos y a $20 \mathrm{rpm}$ durante 20 minutos. Una vez finalizada la agitación, la solución se dejó sedimentar durante 30 minutos. La efectividad del tratamiento se estableció midiendo los parámetros de turbidez y SST al agua final y calculando el porcentaje de remoción para cada uno de ellos. Todos los ensayos se efectuaron por duplicado y la desviación estándar calcula fue inferior al $5 \%$ en todos los casos. 
La turbidez se determinó utilizando un equipo Turbiquant 1500T, MERCK. Las medidas de conductividad se efectuaron con un conductímetro Cond 3210, Tetracon 325, WTW. Los parámetro de SST, DBO y DQO se determinaron de acuerdo a normas detalladas en el Standar Methods for the Examination of Water and Wastewater (Eaton et al., 2005). Con el fin de determinar la composición química de los lodos generados en el proceso de beneficio, Las medidas de FRX se realizaron en un espectrómetro THERMO, modelo Optim'X. Las medidas de Área superficial BET se llevaron a cabo en un equipo Gemini V, Micromeritics. Las muestras se desgasificaron bajo condiciones de vacío y flujo de $\mathrm{N}_{2}$, a una temperatura de $100{ }^{\circ} \mathrm{C}$ durante 12 horas

\section{RESULTADOS Y DISCUSIÓN}

\section{Tratamiento fisicoquímico de las aguas residuales}

En la Tabla 2 se presentan los resultados de la caracterización fisicoquímica efectuada a las aguas residuales provenientes de una industria local de cerámicos. El decreto 1594 de 1984 reporta que para potabilizar aguas la turbidez deberá ser menor o igual a 10 UJT (Unidades Jackson de Turbiedad) o su equivalente a 190 NTU según el artículo 39 del decreto 1594 de 1984.

Tabla 2: Caracterización fisicoquímica del agua residual.

\begin{tabular}{ccc}
\hline Parámetro & Valor & Decreto 1594 \\
\hline SST & $27.11 \mathrm{mg} / \mathrm{L}$ & $80 \%$ remoción \\
Turbiedad agitada & $>1000 \mathrm{UTN}$ & No reporta \\
Turbiedad reposada * & $803 \mathrm{UTN}$ & No reporta \\
Conductividad & $343 \mu \mathrm{S} / \mathrm{cm}$ & No reporta \\
DBO5 & $61.2 \mathrm{mg} / \mathrm{L} \mathrm{O}_{2}$ & $80 \%$ remoción \\
DQO & $90 \mathrm{mg} / \mathrm{L} \mathrm{O}_{2}$ & No reporta \\
\hline
\end{tabular}

El agua residual analizada posee una alta concentración de SST, así como un elevado valor de turbiedad, tanto agitada como reposada. Los valores de DBO y DQO son igualmente altos. Los resultados indican que no es deseable verter estas aguas en las corrientes acuáticas municipales sin un tratamiento previo que permita remover los SST y bajar la turbidez del sistema acuoso.

En la Figura 1 se presentan los resultados obtenidos en la evaluación de los dos coagulantes a pH 6 y una concentración de coagulante de $20 \mathrm{mg} / \mathrm{L}$ (Figura 1(a)) y $100 \mathrm{mg} / \mathrm{L}$ (Figura 1(b)). Se observa que a una concentración de $100 \mathrm{mg} / \mathrm{L}$ se obtienen altos porcentajes de remoción de ambos parámetros. Mientras que con el sulfato de aluminio se obtiene una alta remoción de turbiedad $(98.4 \%)$, el polímero permite obtener una alto porcentaje de remoción de SST (99.2\%) Bajo las condiciones indicadas., los resultados obtenidos con ambos coagulantes es destacable.
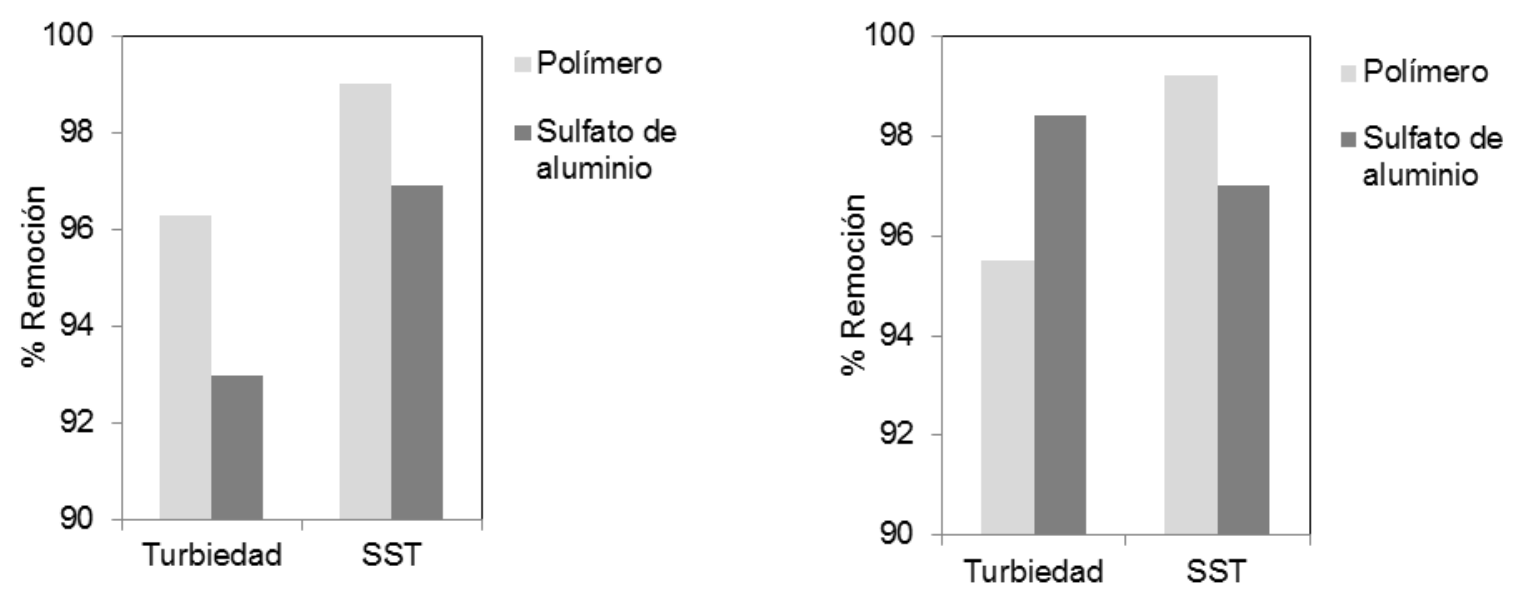

Fig. 1: Porcentaje de Remoción de SST y turbiedad a pH 6 y una dosis de coagulante de: (a) 20 mg/L y (b) $100 \mathrm{mg} / \mathrm{L}$

A una concentración de $20 \mathrm{mg} / \mathrm{L}$ se obtienen porcentajes de remoción significativos. El polímero aniónico permite obtener una remoción de turbiedad cercana al 96\% y del orden del 99\% en los SST, mientras que con el sulfato de aluminio los porcentajes de remoción de turbiedad y SST son $93 \%$ y $96.9 \%$, respectivan (a) . Si bien el porcentaje de remoción de la turbidez del sistema acuoso decrece con respecto 
al obtenido con una dosis de coagulante de $100 \mathrm{mg} / \mathrm{L}$, cabe destacar que sigue siendo un porcentaje lo suficientemente alto como para garantizar una correcta disposición de las aguas finales de la industria a una concentración que es 5 veces inferior.

A un $\mathrm{pH}$ de 6 y una dosis de coagulante de $20 \mathrm{mg} / \mathrm{L}$ es evidente que el mejor desempeño en el proceso de clarificación de las aguas tratadas corresponde al polímero aniónico. Esta situación puede ser explicada teniendo en cuenta que, efectivamente, este tipo de ayudantes del proceso de coagulación se caracterizan por su alta efectividad a bajas concentraciones. El hecho de poseer grupos activos distribuidos a lo largo de su cadena le confiere a este tipo de materiales una alta afinidad por las superficies sólidas. El alto porcentaje de remoción de los SST $(99 \%)$ es, en efecto, resultado de la interacción entre las partículas suspendidas y el coagulante. Se ha establecido también que los floculantes poliméricos tienden a mejorar la cohesión de los flóculos y a incrementar la velocidad de sedimentación, lo cual incide de forma positiva en la eficiencia del proceso de coagulación-floculación.

En la Figura 2 se muestran los resultados obtenidos en la prueba de jarras a un pH de 9 y una concentración de coagulante de $20 \mathrm{mg} / \mathrm{L}$ (Figura 2(a)) y $100 \mathrm{mg} / \mathrm{L}$ (Figura 2(b)).
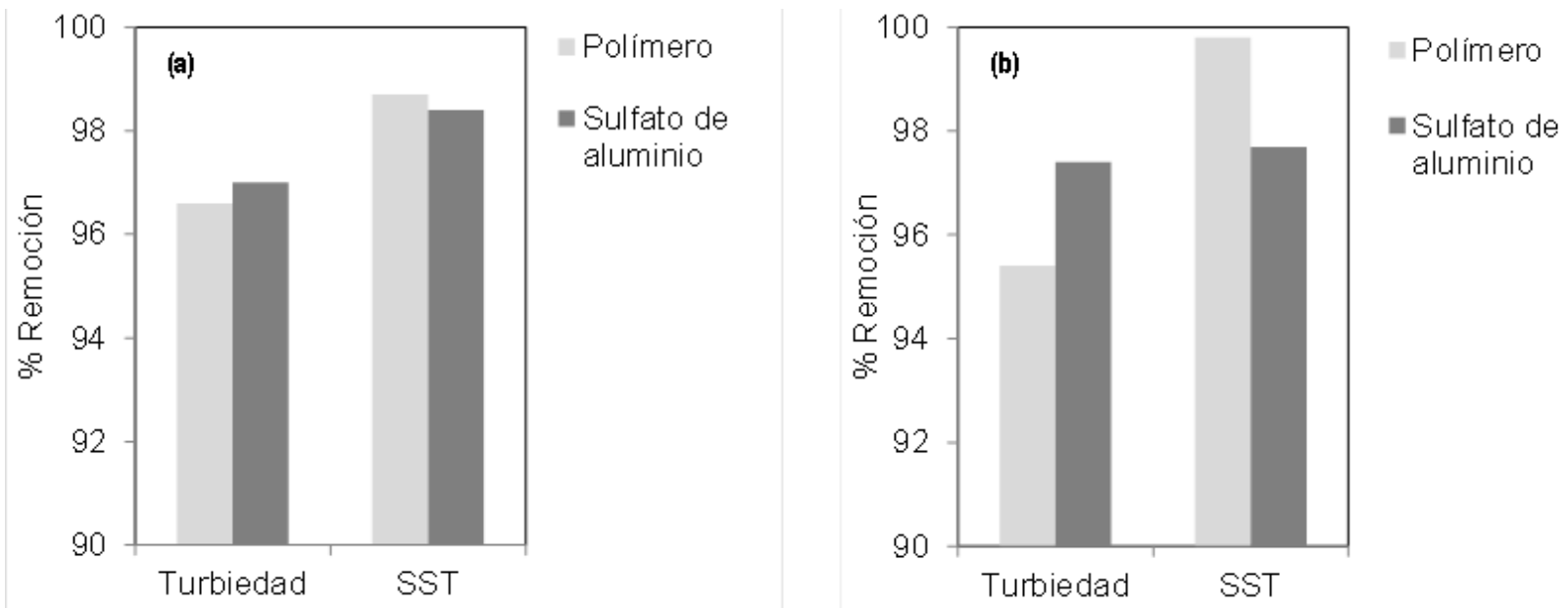

Fig. 2: Porcentaje de Remoción de SST y turbiedad a pH 9 y una dosis de coagulante de: (a) 20 mg/L y (b) $100 \mathrm{mg} / \mathrm{L}$.

Los resultados obtenidos a un pH de 9 muestran que el proceso de remoción de SST y turbidez sigue siendo altamente efectivo. A una concentración de $20 \mathrm{mg} / \mathrm{L}$ el polímero exhibe una remoción de SST del $98.7 \%$ y de turbidez del $96.6 \%$, en tanto que con el sulfato de aluminio los porcentajes de remoción de SST y de turbidez fueron $98.4 \%$ y $97 \%$, respectivamente. Es claro que el desempeño de ambos coagulantes bajo estas condiciones es comparable. A una concentración de $100 \mathrm{mg} / \mathrm{L}$ la mayor diferencia reside en el porcentaje de remoción de SST con el polímero, cuyo valor ascendió a un $99.8 \%$. La remoción de turbidez fue ligeramente inferior para el polímero, cercana al $95.5 \%$, mientras que con el sulfato de aluminio se alcanzó un valor de $97.4 \%$ para este parámetro.

Comparando los resultados obtenidos con otros reportados en la literatura, es posible afirmar que estos son altamente satisfactorios. Por ejemplo, Guan y colaboradores (Guan et al., 2005) estudiaron la posibilidad de reutilizar lodos cargados de aluminio con el fin de mejorar la remoción de contaminantes particulados de aguas residuales de una planta de tratamiento ( $S S$ entre $236-356 \mathrm{mg} / \mathrm{L}, \mathrm{pH}$ entre 7.2-7.8)). Los experimentos efectuados se llevaron a cabo mediante la prueba de Jarras. Se encontró que las eficiencias de eliminación tanto de los SS como de la DQO mejoraron en un $20 \%$ y un $15 \%$, respectivamente, debido a la introducción de dichos lodos, lo cual fue atribuido principalmente a la eliminación de partículas con un tamaño de entre 48 a $200 \mu \mathrm{m}$. Se estableció que la dosis apropiada de lodos era de $18-20 \mathrm{mg} \mathrm{Al} / \mathrm{L}$ y que al aumentar la velocidad de mezclado rápido o reducir el tamaño de flóculo del lodo cargado de aluminio, la eliminación de la DQO podría mejorarse aún más.

Por otra parte, Rivas y colaboradores (Rivas et al, 2010) evaluaron el tratamiento de aguas residuales provenientes de una industria de quesos (SST entre 1628-4780 mg/L, turbiedad entre 1331-2004 UTN), utilizando tres coagulantes diferentes: $\mathrm{FeSO}_{4}, \mathrm{Al}_{2}\left(\mathrm{SO}_{4}\right)_{3}$ y $\mathrm{FeCl}_{3}$. Cuando se utilizó $\mathrm{FeSO}_{4}$, las condiciones óptimas obtenidas fueron $250 \mathrm{ppm}$ de la sal a un pH de 8.5, obteniéndose un $50 \%$ y $60 \%$ de reducción de la DQO y de la DBO, respectivamente. Con el sulfato de aluminio se lograron porcentajes de reducción de estos parámetros ligeramente menores, no obstante, la dosis requerida fue significativamente mayor (1000 ppm). Cuando se evaluó la sal de cloruro férrico se obtuvieron resultados similares a los reportados con el 
sulfato ferroso, es decir, a una concentración de 250 ppm se eliminó la DBO y la DQO en el intervalo comprendido entre $40-60 \%$, dependiendo de las condiciones de evaluación. El lodo formado en el proceso de coagulación-floculación mostró aceptables propiedades de sedimentación.

Beltrán-Heredia y Sánchez-Martín (Beltrán-Heredia y Sánchez-Martín, 2009) evaluaron un nuevo coagulante y floculante basado en taninos (TANFLOC) para el tratamiento de aguas residuales urbanas (SS $100 \mathrm{mg} / \mathrm{L}$, turbidez 82.5 UTN). TANFLOC mostró una alta efectividad en la eliminación de la turbidez (casi $100 \%$, dependiendo de la dosis) y alrededor de un $50 \%$ en la eliminación de la $\mathrm{DBO}_{5}$ y de la DQO, eficacia que fue comparable a la del sulfato de aluminio. El proceso de coagulación y floculación no dependió de la temperatura, y la velocidad de agitación óptima y el tiempo fueron establecidos en $40 \mathrm{rpm}$ durante 30 min.

Los resultados obtenidos en este trabajo claramente indican que el proceso de coagulación-floculación fue sumamente eficiente para la remoción de SST y turbidez de las aguas analizadas, bajo las condiciones evaluadas. En este contexto, la decisión de cual coagulante utilizar y bajo qué condiciones, debe apoyarse en las siguientes consideraciones: $\mathrm{pH}$ del proceso (entre más cercano a $\mathrm{pH}$ neutro mejor), dosis de coagulante (mínima) y costos del proceso.

Es claro que un $\mathrm{pH}$ de 6 y una dosis de coagulante de $20 \mathrm{mg} / \mathrm{L}$ garantizan una alta remoción de los parámetros evaluados, la cual no difiere de manera significativa con la obtenida bajo condiciones de proceso más drásticas. En cuanto al tipo de coagulante, el sulfato de aluminio es un material de fácil consecución, económico y que mostró una alta efectividad para tratar las aguas de la industria local de cerámicos. No obstante, tiene la desventaja de producir grandes cantidades de lodos que contienen insolubles como el hidróxido de aluminio. Por el contrario, los ayudantes de coagulación de tipo polimérico se caracterizan por su baja producción de lodos (sin contenido de insolubles), por ser efectivos en un amplio rango de $\mathrm{pH}$ (usualmente no modifican el $\mathrm{pH}$ del sistema), además de mejorar la resistencia de los flóculos frente a las fuerzas de cizalladura y de exhibir altas velocidades de sedimentación (Tyagi et al., 2010). Estas propiedades son de vital importancia en aras de obtener aguas óptimamente clarificadas.

Teniendo en consideración la posibilidad de reutilizar el lodo generado en el proceso de beneficio de arcillas y caolines, y la discusión anteriormente expuesta, se recomienda implementar el proceso de fisicoquímico de tratamiento de las aguas con el polímero aniónico bajo las condiciones óptimas ( $\mathrm{pH} 6,20 \mathrm{mg} / \mathrm{L})$. Se recomienda además, efectuar ensayos adicionales a dosis más bajas del mismo, pues es probable que su comportamiento siga siendo destacable a concentraciones inferiores, dada su naturaleza química y las propiedades del mismo. Implementar un proceso de esta naturaleza en una industria de este tipo es relativamente sencillo, y la efectividad del tratamiento puede mejorarse aún más complementándolo con una serie de sedimentadores y decantadores o de ser posible, con la utilización de un sistema de filtroprensado para remover simultáneamente los lodos del proceso y clarificar sustancialmente las aguas, con lo cual la dosis de coagulante a utilizar sería aún menor.

\section{Alternativas de valorización para los lodos residuales}

En la Tabla 3 se muestran los resultados obtenidos por FRX para tres materias primas diferentes y en la Tabla 3 se reportan los resultados de FRX para la muestra de lodo, todos provenientes de una industria local de beneficio de arcillas y caolines. Los resultados se expresan como porcentaje en peso.

Tabla 3: Caracterización por FRX de tres diferentes arcillas utilizadas como materia prima en el proceso de beneficio.

\begin{tabular}{lccc}
\hline \multicolumn{1}{c}{$\underline{\text { Oxido }}$} & $\underline{M 1}$ & $\underline{M 2}$ & $\underline{M 3}$ \\
\hline$\underline{\text { Óxido de Aluminio }\left(\mathrm{Al}_{2} \mathrm{O}_{3}\right)}$ & $\underline{42.02}$ & $\underline{\underline{20.36}}$ & $\underline{35.96}$ \\
\hline Óxido de Silicio $\left(\mathrm{SiO}_{2}\right)$ & $\underline{36.17}$ & $\underline{65.49}$ & $\underline{49.00}$ \\
\hline Óxido de Hierro $\left(\mathrm{Fe}_{2} \mathrm{O}_{3}\right)$ & 0.57 & 1.48 & 0.50 \\
Óxido de Potasio $\left(\mathrm{K}_{2} \mathrm{O}\right)$ & 0.10 & 0.59 & 0.15 \\
Óxido de Titanio $\left(\mathrm{TiO}_{2}\right)$ & 1.45 & 0.96 & 1.30 \\
Óxido de Magnesio $(\mathrm{MgO})$ & 0.08 & 0.32 & 0.08 \\
Óxido de Manganeso $(\mathrm{MnO})$ & 0.02 & 0.02 & 0.01 \\
Óxido de Cromo $\left(\mathrm{Cr}_{2} \mathrm{O}_{3}\right)$ & 0.04 & 0.04 & 0.04 \\
Óxido de Calcio $\left(\mathrm{CaO}^{2}\right)$ & 0.00 & 0.10 & 0.00 \\
Pérdidas por calcinación (LOI) & 18.89 & 10.05 & 13.36 \\
\hline
\end{tabular}


De acuerdo a la Tabla 3, las muestras están constituidas principalmente por óxido de aluminio y óxido de silicio, como es de esperarse en materiales tipo arcillas. La composición de la muestra M3 es típica de las arcillas caoliníticas. La composición del lodo residual, de acuerdo a la Tabla 4, muestra un alto contenido de óxido de silicio y en una menor proporción, óxido de aluminio, con trazas de algunos óxidos inorgánicos. No se observa la presencia de metales pesados, ni de otros tipos de impurezas que puedan afectar la calidad del lodo residual, limitando sus posibles aplicaciones.

Tabla 4: Caracterización por FRX para el lodo generado en el proceso de beneficio.

\begin{tabular}{lc}
\hline \multicolumn{1}{c}{ Oxido } & Lodo \\
\hline Óxido de Aluminio $\left(\mathrm{Al}_{2} \mathrm{O}_{3}\right)$ & 25.21 \\
Óxido de Silicio $\left(\mathrm{SiO}_{2}\right)$ & 58.93 \\
Óxido de Hierro $\left(\mathrm{Fe}_{2} \mathrm{O}_{3}\right)$ & 0.57 \\
Óxido de Potasio $\left(\mathrm{K}_{2} \mathrm{O}\right)$ & 1.30 \\
Óxido de Titanio $\left(\mathrm{TiO}_{2}\right)$ & 0.70 \\
Óxido de Magnesio $(\mathrm{MgO})$ & 0.45 \\
Óxido de Manganeso $(\mathrm{MnO})$ & 0.02 \\
Óxido de Cromo $\left(\mathrm{Cr}_{2} \mathrm{O}_{3}\right)$ & 0.04 \\
Óxido de Calcio $(\mathrm{CaO})$ & 0.80 \\
Óxido de Sodio $\left(\mathrm{Na}_{2} \mathrm{O}\right)$ & 0.28 \\
Pérdidas por calcinación $(\mathrm{LOI})$ & 10.08 \\
\hline
\end{tabular}

El área superficial del lodo residual es de $23 \mathrm{~m}^{2} / \mathrm{g}$, mientras que el valor del área superficial para la mezcla de materias primas que se utiliza en la fabricación de productos cerámicos es de $38 \mathrm{~m}^{2} / \mathrm{g}$. Esta diferencia puede ser atribuida a la menor presencia de óxido de aluminio en el lodo. Dado que en la fabricación de productos cerámicos, las arcillas contribuyen con aproximadamente un 95\% del área superficial de la mezcla compuesta, en el comportamiento coloidal de las arcillas domina la reología de los productos cerámicos. Si bien el lodo residual presenta un área superficial menor, es posible incorporarlo en proporciones adecuadas de forma tal que el área superficial de la mezcla de materias primas se preserve 0 en su defecto, no se modifique de forma significativa y no se alteren las propiedades requeridas para la fabricación de las piezas cerámicas.

De acuerdo a la naturaleza química del lodo residual se proponen las siguientes opciones como alternativas para valorizarlo:

Reutilizarlo en el proceso: Esta posibilidad es factible dado que el lodo residual contiene una composición rica en sílice y alúmina, lo cual se evidenció en los análisis de FRX realizados. No obstante, la pasta residual presenta una coloración fuerte que eventualmente podría otorgar puntos o algún tipo de coloración a las piezas cerámicas fabricadas. Es necesaria la realización de pruebas con el fin de determinar la carga de lodo que puede adicionársele a la materia prima con el fin de no comprometer parámetros de calidad.

Fabricar un producto de menor precio y menor calidad (gama baja): Se propone la fabricación de piezas cerámicas de menor calidad (línea económica), que básicamente se vería afectada en su apariencia externa, más no en su composición estructural. Esta opción podrís ser sumamente atractiva para las empresas de beneficio de arcillas para producir productos cerámicos, debido a que usualmente descartan el lodo que se produce en el proceso.

Implementar procesos de secado, molienda y posterior caracterización: El lodo una vez sometido a un proceso de secado, molienda y caracterización, puede ser utilizado como material de construcción de ladrillos, tejas, materiales refractarios o como carga en la fabricación de cemento. Es una de las alternativas más viables ya que es un producto óptimo para ser usado como material de carga en la industria del cemento remplazando arenas, y también en la fabricación de refractarios de construcción, en los cuales el aspecto externo no es relevante, más si lo es su integridad estructural.

Fabricación de plafones, inodoros o implementos especiales de cerámica: Esta opción es altamente viable puesto que estos artículos no presentan un estricto control de calidad en cuanto a su aspecto externo. Específicamente, en el caso de los inodoros no existe ningún inconveniente debido a que estos usualmente vienen en diferentes tonalidades, haciendo menos evidente cualquier imperfección o problema de calidad en cuanto al color. Nuevamente cabe resaltar que la composición estructural es primordial. 
Estas opciones se proponen con fundamente en los resultados de caracterización del lodo residual, en la experiencia de los autores y en los resultados obtenidos por diferentes investigadores en el área. Así, Toya y colaboradores (Toya et al, 2004) prepararon materiales vitrocerámicos tipo $\mathrm{CaO}-\mathrm{MgO}-\mathrm{Al}_{2} \mathrm{O}_{3}-\mathrm{SiO}_{2}$, a partir de desechos generados en el proceso de refinación de arcillas tipo caolín (Kira). Los residuos fueron calcinados a $1350{ }^{\circ} \mathrm{C}$ y posteriormente enfriados en agua para obtener vidrios. Mediante procesos de molienda y tamizado se ajustó el tamaño de partícula (<100 mallas). Las pruebas de durabilidad química en ácidas y bases mostraron excelentes resultados, razón por la cual se concluyó que estos desechos son buenos candidatos para ser utilizados como materiales de construcción, baldosas, etc.

Adicionalmente, Junkes y colaboradores (Junkes et al., 2011) evaluaron residuos provenientes de procesos de clarificación y filtración de aguas, arcillas de desecho, entre otros, con el propósito de establecer su posible uso como materia prima en la manufactura de baldosas cerámicas. Los investigadores establecieron cuatro diferentes formulaciones, de acuerdo al diagrama de fases del sistema $\mathrm{SiO}_{2}-\mathrm{Al}_{2} \mathrm{O}_{3}-\mathrm{K}_{2} \mathrm{O}$. Los materiales resultantes fueron clasificados como altamente plásticos de acuerdo a su índice de plasticidad. Se concluyó que los desechos evaluados, al igual que las formulaciones desarrolladas, representan una buena alternativa para la fabricación de las piezas cerámicas.

Rauup-Pereira y colaboradores (Rauup-Pereira et al., 2006) estudiaron desechos industriales y subproductos basados en el sistema $\mathrm{SiO}_{2}-\mathrm{Al}_{2} \mathrm{O}_{3}-\mathrm{CaO}$, con el fin de establecer su viabilidad para fabricar productos cerámicos de interés industrial. Los resultados mostraron que las mezclas efectuadas son promisorias para obtener materiales tipo cemento y refractarios. Finalmente, Menezes y colaboradores (Menezes et al., 2008) caracterizaron desechos provenientes de industrias de procesamiento de caolín y establecieron la viabilidad de utilizarlos como materia prima en la producción de productos cerámicos porosos. La caracterización efectuada mostró que los desechos están conformados por cuarzo, caolinita y mica, y que formulaciones hasta con un $66 \%$ de contenido de desechos pueden ser utilizadas en la producción de cerámicos con porosidad superior al $40 \%$ y resistencia mecánica cercana a $70 \mathrm{MPa}$.

Si bien la composición química del lodo hace prever que las aplicaciones propuestas son altamente factibles, es necesaria la evaluación de las propiedades fisicoquímicas y mecánicas del material en las aplicaciones propuestas, con el fin de garantizar que se cumplan las condiciones mínimas de calidad para el eventual desarrollo de los diferentes productos.

\section{CONCLUSIONES}

Se llevó a cabo el tratamiento fisicoquímico de las aguas residuales provenientes de una industria local de beneficio de arcillas y caolines. Los porcentajes de remoción de SST y turbidez alcanzados en las pruebas de jarras mostraron una alta eficiencia para tratar las aguas residuales. Los resultados obtenidos a un $\mathrm{pH}$ de 6 y una dosis de coagulante de $20 \mathrm{mg} / \mathrm{L}$ fueron remarcables, especialmente con el polímero aniónico, con el cual se obtuvo un porcentaje de remoción de SST cercano al $99 \%$ y de la turbidez del orden de $96 \%$. Con el sulfato de aluminio se obtuvieron porcentajes de remoción de ambos parámetros ligeramente inferiores. Se recomienda la utilización del polímero aniónico bajo las condiciones indicadas, dada su baja tendencia a la producción de lodos, a la calidad de los mismos (no insolubles), la calidad de los flóculos generados y la alta velocidad de sedimentación. Es deseable examinar el desempeño del polímero aniónico a concentraciones más bajas, con el fin de disminuir los costos del proceso.

La caracterización química de la materia prima y del lodo generado en el proceso de beneficio de arcillas y caolines, así como los resultados obtenidos por diferentes investigadores en la materia, permitió explorar algunas alternativas viables para valorizar los lodos: reutilizarlos en el proceso (carga baja), fabricar un producto de menor calidad, implementar procesos de secado, molienda y caracterización para determinar la viabilidad de fabricar ladrillos, tejas, refractarios, implementos especiales de cerámica que no requieran altos controles de calidad en su apariencia externa y como material de carga en la industria cementera.

\section{AGRADECIMIENTOS}

Los autores agradecen a la Universidad de Antioquia - Comité para el Desarrollo de la Investigación (CODI) y a Colciencias - Patrimonio Autónomo Fondo Nacional de Financiamiento para la Ciencia, la Tecnología y la Innovación, Francisco José de Caldas.

\section{REFERENCIAS}

Alvarez, M., Ardila, H., Mosquera, D, El Impacto Ambiental de la Pequeña y Mediana Empresa en los Recursos Hídricos de Colombia, Agua Latinoamérica: 1(3), 27-29, (2001). 
Aragonés-Beltrán, P., J.A. Mendoza-Roca, A. Bes-Piá, M. García-Melón y E. Parra-Ruiz, Application of multicriteria decision analysis to jar-test results for chemicals selection in the physical-chemical treatment of textile wastewater, Journal of Hazardous Materials: 164(1), 288-295 (2009).

Barrera-Díaz, C., I. Linares-Hernández, G. Roa-Morales, B. Bilyeu y P. Balderas-Hernández, Removal of biorefractory compounds in industrial wastewater by chemical and electrochemical pretreatments, Ind. Eng. Chem. Res: 48(3), 1253-1258 (2009).

Beltrán-Heredia, J. y J. Sánchez-Martín, Municipal wastewater treatment by modified tannin flocculant agent, Desalination: 249(1), 353-358 (2009).

Bouyer, D., R. Escudié y A. Liné, Experimental analysis of hydrodynamics in a jar-test, Trans IChemE, Part B, Process Safety and Environmental Protection: 83(B1), 22-30 (2005).

Clark, T. y T. Stephenson, Development of a jar-testing protocol for chemical phosphorous removal in activated sludge using statistical experimental design, Water Research: 33 (7), 1730-1734 (1999).

Eaton, A.D. y otros tres autores, Standard Methods for the Examination of Water and Wastewater: Inc. 5Day BOD Test 5210 B, Pág. 5-2, Ed. United Book Press, 21 $1^{\text {st }}$ Edition, USA (2005).

Eaton, A.D. y otros tres autores, Standard Methods for the Examination of Water and Wastewater: Inc. Closed Reflux, Colorimetric Method 5220 D, Pág. 5-18, Ed. United Book Press, $21^{\text {st }}$ Edition, USA (2005).

Eaton, A.D. y otros tres autores, Standard Methods for the Examination of Water and Wastewater: Inc. Total Suspended Solids Dried at 103-105 을, Pág. 2-58, Ed. United Book Press, 21 ${ }^{\text {st }}$ Edition, USA (2005).

Environmental Protection Agency, BAT Guidance Note on Best Available Techniques for the Manufacture of Ceramic Products and Industrial Diamonds (2012), http://www.epa.ie/pubs/advice/bat/batguidancenotefortheceramicdiamondsector.html. Acceso: 12 de agosto (2013).

Guan, X.-H., G.H. Chen y C. Shang, Re-use of water treatment works sludge to enhance particulate pollutant removal from sewage, Water Research: 39(15), 3433-3440 (2005).

Ji, Y., Q. Lu, Q. Liu y H. Zeng, Effect of solution salinity on settling of mineral tailings by polymer flocculants, Colloids and Surfaces A: Physicochem. Eng. Aspects: 430, 29-38 (2013).

Junkes, J.A., M.A. Carvalho, A.M. Segadães y D. Hotza, Ceramic tile formulations from industrial waste, Interceram: 60(1), 36-41 (2011).

Kim, C.S., K.M. Wilson y J.J. Rytuba, Particle-size dependence on metal(loid) distributions in mine wastes: Implications for water contamination and human exposure, Applied Geochemistry: 26(4), 484-495 (2011).

Koivunen, J., A. Siitonen y $\mathrm{H}$. Heinonen-Tanski, Elimination of enteric bacteria in biological-chemical wastewater treatment and tertiary filtration units, Water Research: 37(3), 690-698 (2003).

Li, J., S. Jiao, L. Zhong, J. Pan y Q. Ma, Optimizing coagulation and flocculation process for kaolinite suspension with chitosan, Colloids and Surfaces A: Physicochem. Eng. Aspects: 428,100-110 (2013).

McCarthy, J.F. y J.M. Zachara, Subsurface transport of contaminants, Environ. Sci. Technol: 23 (5), 496-502 (1989).

Menezes, R.R., M.I. Brasileiro, L.N.L. Santana, G.A. Neves, H.L. Lira y H.C. Ferreira, Utilization of kaolin processing waste for the production of porous ceramic bodies, Waste Management \& Research: 26(4), 362368 (2008).

Miller, S.M., E.J. Fugate, V.O. Craver, J.A. Smith y J.B. Zimmerman, Toward understanding the efficacy and mechanism of Opuntia spp. as a natural coagulant for potential application in water treatment, Environ. Sci. Technol: 42(12), 4274-4279 (2008).

Moreno, H.A, C.V. Droppelmann y M.E. Verdejo, Evaluación de Carbón Activado Producido a partir de Lodo Generado en una Planta de Tratamiento de Aguas Servidas, Inf. Tecnol: 17(3), 9-14 (2006). 
Raupp-Pereira, F., D. Hotza, A.M. Segadães y J.A. Labrincha, Ceramic formulations prepared with industrial wastes and natural sub-products, Ceramics International: 32, 173-179 (2006).

Rivas, J., A.R. Prazeres, F. Carvalho y F. Beltrán, Treatment of cheese whey wastewater: combined coagulation-flocculation and aerobic biodegradation, J. Agric. Food Chem: 58(13), 7871-7877 (2010).

Souza, L.P.de F., Mansur, H.S. Production and characterization of ceramic pieces obtained by slip casting using powder wastes. Journal of Materials Processing Technology 145: 14-20, 2004.

Toya, T., Y. Tamura, Y. Kameshima y K. Okada, Preparation and properties of $\mathrm{CaO}-\mathrm{MgO}-\mathrm{Al}_{2} \mathrm{O}_{3}-\mathrm{SiO}_{2}$ glass-ceramics from kaolin clay refining waste (Kira) and dolomite, Ceramics International: 30(6), 983-989 (2004).

World Health Organization, UN-Water Global Analysis and Assessment of Sanitation and Drinking-Water Assessment: The challenge of extending and sustaining services (2012), http://www.who.int/water_sanitation_health/publications/glaas_report_2012/en/index.html. Acceso: 1 de agosto (2013).

Tyagi, V.K., A.A. Khan, A.A. Kazmi y A.K. Chopra, Enhancement of coagulation flocculation process using anionic polymer for the post treatment of UASB reactor effluent, Separation Science and Technology: 45(5), 626-634 (2010). 